\title{
Exploiting evolution to treat drug resistance: Combination therapy and the double bind
}

\author{
David Basanta1, Robert A. Gatenby ${ }^{1}$, and Alexander R. A. Anderson ${ }^{1}$ \\ ${ }^{1}$ Integrated Mathematical Oncology, H. Lee Moffitt Cancer Center and Research Institute, Tampa, \\ FL 33612, USA
}

\section{Abstract}

Although many anticancer therapies are successful in killing a large percentage of tumour cells when initially administered, the evolutionary dynamics underpinning tumour progression mean that often, resistance is an inevitable outcome. Research in the field of ecology suggests that an evolutionary double bind could be an effective way to treat tumours. In an evolutionary double bind two therapies are used in combination such that evolving resistance to one leaves individuals more susceptible to the other. In this paper we present a general evolutionary game theory framework of a double bind to study the effect that such approach would have in cancer. Furthermore we use this mathematical framework to understand recent experimental results that suggest a synergistic effect between a p53 cancer vaccine and chemotherapy. Our model recapitulates the latest experimental data and provides an explanation for its effectiveness based on the commensalistic relationship between the tumour phenotypes.

\section{Keywords}

Evolutionary Game Theory; Evolutionary Double Bind; Mathematical Modelling;

Immunotherapy; Chemotherapy; Combination therapy; comensalism

\subsection{Introduction}

Cancer is an evolutionary disease [1]. One of the most negative aspects of its evolutionary nature is that tumours can evolve resistance to anti-cancer treatments. It has recently been suggested that we should consider therapies that exploit evolutionary dynamics rather than fail because of them. One possible evolutionary enlightened therapy would be to exploit an evolutionary double bind. In an evolutionary double bind a species can be preyed upon at least by two species of predators. Under these circumstances if one of the predator species selects for an adaptation that makes the preys safer from that predator but more susceptible to a different species of predators then that situation can be described as an evolutionary double bind. In the context of cancer, where treatments could be seen as predators and tumour cells as preys, an evolutionary double bind can be understood as a combination therapy in which adaptation to it represents a significant fitness cost [2]. This cost could represent a number of things, such as a lower proliferation rate to minimise exposure to drugs that target mitosis, the reallocation of resources away from proliferation in order to obtain resistance or switching to alternative less efficient pathways that are not targeted. Combination therapies are those in which more than one treatment is administered to a patient with the aim to hinder emergence of resistant types. Unfortunately not all combination therapies work synergistically since certain tumour cell adaptations result in

\footnotetext{
2david@CancerEvo.org.
} 
resistance to more than one treatment. Yet combination therapies have the potential to hinder the evolution of resistance since they can be designed so that tumours need to adapt to each treatment independently and resistance to one treatment could make resistance to the other more difficult. In this sense combination therapies are potentially an ideal approach to facilitate an evolutionary double bind, where adaptation to one treatment would leave resistant tumour cells more susceptible to another treatment than the original population.

Its generally well accepted now that cancer consists of multiple populations that are phenotypically (as well as genotypically) distinct. This inherent heterogeneity that all cancers appear to exhibit is one of the central reasons why resistance emerges in the first place. Since a mono-clonal population made of phenotypically identical cells would be far easier to target therapeutically than one that was made of a heterogeneous mix of phenotypes. Also, such heterogeneity could allow for preexisting resistance as opposed to de novo resistance. Regardless of the precise mechanism of resistance, reducing heterogeneity in a cancer population should make it a more treatable disease. However, we must be cautious as treatments that manipulate this heterogeneity may select more aggressive phenotype rather than weaker ones [3]. If it is possible to exploit an evolutionary double bind we will naturally circumvent this problem.

In this paper we introduce a general theoretical framework to study the potential of evolutionary double binds based on combination therapies as a cancer treatment. This framework uses evolutionary game theory (EGT), a mathematical tool that has been traditionally used in evolutionary ecology [4] and more recently in mathematical oncology $[5,6,7,8,9,10]$. This modelling tool has potential to provide insights into the evolutionary dynamics of cancer when a combination therapy is used. Importantly EGT can help explore the role of tumour cell interactions in the emergence of resistance. More specifically we have utilized an EGT model to understand how chemotherapy can improve the efficacy of a p53 vaccine as has been reported by Antonia, Gabrilovich and colleagues $[11,12]$.

\subsection{A general framework to study an evolutionary double bind}

Game Theory is a mathematical framework introduced by Von Neumann and Morgenstern in order to better understand how the interactions between players affect the outcome of the game [13]. Although game theory continues to be a vibrant area of study in the fields of economy and sociology, a variant known as evolutionary game theory has been successful in the field of evolutionary biology. Evolutionary game theory, introduced by Maynard Smith in the seventies [4] studies games where the goal is long term proliferation, the players are individuals in the population and the strategies are not part of a set from which a player can chose one, but its hardcoded in the form of a phenotype. In this game we consider three different populations and two different treatments. For simplicity the treatments will be referred to as $\mathrm{A}$ and $\mathrm{B}$. The populations are $\mathrm{S}$, susceptible to both treatments, $R_{A}$ that is completely resistant to treatment $\mathrm{A}$ but susceptible to treatment $\mathrm{B}$ and $R_{B}$ that is resistant to treatment $\mathrm{B}$ and susceptible to treatment $\mathrm{A}$. The cost of resistant is determined by $c_{A}$ and $c_{B}$ and is assumed to be a fixed cost affecting the fitness of the phenotypes $R_{A}$ and $R_{B}$ respectively. The cost of treatment A to those phenotypes that are nonresistant (i.e.: $S$ and $R_{B}$ ) is a function of time, $d_{A}$, that is a given constant value when the drug is applied and zero when it is not. The drug B has been modelled in the same way but affecting only $S$ and $R_{A}$. The total population is made of these three phenotypes with a proportion $p_{A}$ of $\operatorname{drug} \mathrm{A}$ resistant cells, $p_{B}$ of drug $\mathrm{B}$ resistant cells and the rest made of susceptible cells. Under these assumptions, the following equations represent the fitness of each of the subpopulations: 


$$
\begin{aligned}
& W\left(R_{A}\right)=1-c_{A}-\alpha d_{B}(t) \\
& W\left(R_{B}\right)=1-c_{B}-\beta d_{A}(t) \\
& W(S)=1-d_{A}(t)-d_{B}(t)
\end{aligned}
$$

Where $\alpha$ and $\beta$ represent the extra cost of a resistant phenotype being subjected to a different drug. These constants capture the double bind aspect of the model, since a phenotype resistant to one drug can be more susceptible to the other.

Given that the average fitness $(W \overline{)}$ in the population can be defined as:

$$
\overline{\mathrm{W}}=p_{A} * W\left(R_{A}\right)+p_{B} * W\left(R_{B}\right)+\left(1-p_{A}-p_{B}\right) * W(S)
$$

the proportion $p$ of a given phenotype $i$ in the tumour population at a given time $t$ then will be given by:

$$
p_{t+1}=p_{t} * \frac{W(i)}{\overline{\mathrm{W}}}
$$

This model assumes that interactions between these populations have no impact on the evolution of resistance but arguably, the fitness of each of these three populations could change as a result of those interactions. For instance, assuming that space and nutrients are scarce, a resistant phenotype interacting with susceptible cells will be better off than the same cell interacting with equally resistant cells as in the first case it would have easier access to nutrients and space required for proliferation. This and other hypothetical mechanisms for resistance, like paracrine ones that could affect phenotypes that do not produce them, could be further explored with a matrix based approach. Using a matrix approach we can explore how the interactions between different phenotypes impacts their fitness. The following table enhances the previously described model by describing how interactions between phenotypes influence the susceptibility of cells to treatments and thus potentially improve their fitness:

where $X(t)$ is the extra benefit/cost to $R_{A}$ cells interacting with cells susceptible to drug A when drug $\mathrm{A}$ is being delivered. Correspondingly, $Y(t)$ is the extra benefit/cost for $R_{B}$ when interacting with drug $B$ sensitive cells when drug $B$ is being administered. From this table we can define the fitness equation of each population as follows:

$$
\begin{gathered}
W\left(R_{A}\right)=1-c_{A}-\alpha d_{B}(t)+\left(1-p_{A}\right) X(t) \\
W\left(R_{B}\right)=1-c_{B}-\beta d_{A}(t)+\left(1-p_{B}\right) Y(t) \\
W(S)=1-d_{A}(t)-d_{B}(t)
\end{gathered}
$$

\section{4 p53 cancer vaccine and chemotherapy as an example of an evolutionary double bind}

Cancer immunotherapy is an important field of cancer research that has not yet been able to achieve its real potential. Recent studies by Antonia, Gabrilovich and colleagues [11] 
suggest that a p53 vaccine [14] could have its efficacy improved if administered with chemotherapy. The specific reasons for this are unknown but the authors have recently suggested that the application of drugs such Doxorubicin, Paclitaxel (apoptosis by affecting microtubules) and Cisplatin (death by DNA crosslinking) sensitises the tumour cells to cytotoxic $\mathrm{T}$ cells from the immune system [12].

Antonia et al. performed a clinical trial at the Moffitt cancer center that can, in retrospect, be viewed as a potential test of the double bind concept [11]. The primary goal of the trial was to test a new cancer vaccine that used dendritic cells transduced with the wild-type p53 gene. P53 is a tumour suppressor gene that plays a critical role in cellular growth and differentiation. It is mutated in about $90 \%$ of small cell lung cancers. Mutant p53, unlike the wild type, has a relatively long half life. Thus while the p53 protein is present in low levels in normal cells the mutant protein remains in fairly large concentrations in most SCL cells. Twenty-nine patients with small cell lung cancer (SLC) who failed first line therapy were treated. Evidence of a p53-specific T cell response was observed in $57 \%$ of the vaccinated patients [11]. However, only 1 patient exhibited a partial tumour response (i.e. decrease in the size of a tumour Figure 1.1A). Fortunately, the authors continued to follow the patients after the exited from the study. They found that subsequent treatment with chemotherapy produced a response rate of $62 \%$ (compared to historical controls of less than 5\%).

Interestingly, those patients who exhibited an immunologic response to the vaccine were the more likely to respond to chemotherapy than those that did not (Figure 1.1C). These results indicate that either the adaptive response to the p53 vaccine (perhaps by down-regulation of p53) left the cells more vulnerable to chemotherapy or, alternatively, the chemotherapy rendered the tumour cells more vulnerable to immune attack. In a follow-up study [12] performed in mice, this group found that chemotherapy (specifically Paclitaxel, Cisplatin, and Doxorubicin) increased tumour susceptibility by making tumour cells more permeable to granzyme B, a serine protease that mediates apoptosis by immune cells. These results support a role for chemotherapy as a sensitizer to immunotherapy. However, the rapid decline in immune activity following cessation of the vaccination trial (Figure 1.1D) suggests that other mechanism may play a role.

In the remainder of this paper we will use a more specific version of our general model to understand the evolutionary dynamics that make particular types of combination therapies more effective than others. Using the papers by Antonia, Gabrilovich and colleagues [12, 11, 14] as primary motivation, we will assume three different tumour populations: regular tumour cells that are susceptible to both the p53 vaccine and chemotherapy (S), cells that are resistant to chemotherapy (C) and cells resistant to the p53 vaccine (I). $C$ and $I$ cells have to pay a relatively small cost of resistance $\left(C_{c}\right.$ and $C_{i}$ respectively) which means that $C$ cells do not have to pay any fitness cost as a result of chemotherapy and that $I$ cells do not pay any fitness cost regardless of the concentration of T-cells (whose efficacy is increased by the p53 vaccine). Both chemotherapy and the p53 vaccine represent a fitness cost to the susceptible cells ( $S$ and $I$ in the case of chemotherapy and $S$ and $C$ in the case of the p53 vaccine) only during the time they are applied to the tumour. One important difference between the chemo-resistant population $C$ and the immuno-resistant population $I$ is that we assume that $C$ have an intrinsic type of resistance whereas the $I$ cells evade the immune cells in a paracrine manner which also helps $S$ cells, susceptible to the immune system, interacting with $I$ cells. The modified payoff table is the following:

For simplicity we left $\alpha$ and $\beta$ as constants and equal to 1.1. The treatments $d_{I}(t)$ and $d_{C}(t)$ are Heaviside step functions multiplied by a cost in the range [0:1]. This value and the duration of the step will be variables to characterise the treatment. Under these circumstances the average tumour cell fitness is the following: 


$$
\overline{\mathrm{W}}=p_{C} * W(C)+p_{I} * W(I)+\left(1-p_{C}-p_{I}\right) * W(S)
$$

\subsection{How timing affects the effectiveness of the treatment}

We first validated the mathematical model using the results published by Antonia and colleagues [12]. Figure 1.2 shows the difference in results when chemotherapy is applied first in contrast to when it is applied second. In the first case (1.2 top left) the p53 vaccine is administered first which results in an steady increase of the $I$ population and a dramatic reduction of the $S$ population. After 60 timesteps the p53 vaccine stops being active and chemotherapy is applied after which the tumour almost entirely consists of $C$ cells. In the second case (1.2 top right) the first treatment is chemotherapy which selects for $C$ cells driving the other two populations towards extinction. Figure 1.2 bottom shows the difference between the two therapies in average tumour fitness. To make this comparison the figure shows the average fitness $\bar{W}$ defined by equation 1.4. Here we assume that the average tumour fitness $W$ correlates with the growth of the tumour, based on the fact that this fitness, in the evolutionary sense, is a measure of long term proliferation. By comparing the different impact of the two therapies on tumour fitness we can see why applying chemotherapy first is better (since it results in a lower tumour fitness and slower growth). These results are consistent with those of Gabrilovich [12] and suggest that the application of chemotherapy prior to the p53 vaccination would have a larger impact on the fitness of the tumour and therefore provide a better therapeutic strategy.

The benefit of a simple mathematical model to investigate these dynamics is that we can explore a variety of protocols characterised by order, intensity and duration both quickly and cheaply allowing us to easily predict which protocols would be the most effective. Figure 1.3 shows the results in terms of the average fitness of a tumour cell after iterating the replicator equation for 100 time steps. Darker shades represent a lower fitness. The first row of the figure represents treatments in which the p53 vaccine is applied first (followed by chemotherapy) whereas the second one shows results where chemotherapy has been applied first (followed by the p53 vaccine). Each column represents a different bias towards one of the treatments so the first column shows the first treatment (p53 vaccine in the first row and chemotherapy in the second) applied $25 \%$ of the time whereas the second is applied the remaining $75 \%$ of the time. In each of these scenarios we explored the composition of a tumour at the end of the simulation using different costs of living in an environment with chemotherapy $\left(C_{c}\right)$ and the $\mathrm{p} 53$ vaccine $\left(C_{i}\right)$. Figure 1.3 shows how the predicted average fitness changes as a function of the protocol order. Treatments in which chemotherapy is applied first show that increasing the penalty for chemotherapy and the p53 vaccine has a profound impact on the overall fitness of the tumour. Furthermore, neither chemotherapy nor the p53 vaccine on their own is sufficient to impact the tumour fitness. Results also suggest that applying chemotherapy first, in general, has a higher impact on the tumour fitness. Therefore a higher cost of chemotherapy or a longer exposure to it always results in a less fit tumour.

The situation is different if the p53 vaccine is given before chemotherapy (reversing the order researched by Gabrilovich and colleagues [12]). Here the results suggest that poorer tumour fitness is not necessarily the result of big penalties for chemotherapy and the vaccine (as was the case when chemotherapy is given first). In this case better results are generally obtained when the p53 vaccine is applied for longer period of time.

In order to gain a better understanding of the evolution of the tumour under these treatments we studied in more detail a number of scenarios. Figure 1.4 shows three simulations where 
we kept the cost of cells susceptible to chemotherapy to a constant (0.4) and changed the cost of cells susceptible to the p53 vaccine $(0.2,0.3$ and 0.4$)$. The results show how a high, but not too high, value of the p53 vaccine cost results in a less heterogeneous tumour. On the other hand Figure 1.5 shows three different simulations where the heterogeneity (and ultimate fitness) of the tumour is not affected by the treatments. In this case chemotherapy is applied first for a duration of 25 time steps and relatively low cost $(0.2)$ followed by the p53 vaccine for 75 time steps with varying degrees of cost $(0.2,0.3$ and 0.4$)$.

\subsection{Discussion: reducing heterogeneity}

We have developed a model of the evolutionary double bind and applied this model to explain clinical data resulting from the synergistic combination of chemotherapy and a p53 vaccine. The model is general enough that it could be used to study evolutionary dynamics of double bind in the context of somatic evolution (as well as other evolutionary contexts). The model is also simple requiring only a few parameters that characterise, the cost of resistance to the vaccine and chemotherapy, the cost of susceptibility to either therapy and the cost/benefit of susceptible cells interacting with resistant ones when a given treatment is administered. Our model might not provide the only explanation but the results show that it recapitulates, qualitatively, the observed clinical results when using combination therapies based on immuno- and chemo-therapies. Also, the model can delve deeper into the treatment dynamic and predicts how different scheduling and dosage will impact tumour heterogeneity and fitness. As with all mathematical models, a number of caveats apply: we assume a homogeneously mixed population. We assume only three relevant tumour subpopulations and that these populations do not change over time (e.g. by become more efficient at being resistant). Given the timescales involved (days to weeks) it is unlikely that new genetic mutations would allow the existing populations to change their phenotype significantly.

Despite its simplicity, the model illustrates the importance of understanding the role of cellcell interactions in cancer. Usually these interactions are understood to be competitive in nature but the types of interactions in a tumour can be more complex than that [15]. In the combination therapy example (p53 vaccine and chemotherapy) the relationship between the susceptible and chemo-resistant phenotypes on one hand and the p53 vaccine resistant phenotype on the other seems to correspond to a comensalistic relationship by which the latter provides some degree of protection against the immune system for the former without receiving any reward for that service. That would explain why targeting cells that are sensitive to chemotherapy first is an effective treatment as it disrupts the interactions between the vaccine resistant cells and the rest.

The results coming from the scenarios where the p53 vaccine is applied first are less intuitive. Our results suggest that no single treatment, be it the vaccine or chemotherapy, is sufficient to negatively impact the fitness of the tumour and thus its growth. Although the application of the two therapies is necessary to obtain results only when the vaccine and chemotherapy are administered similar amounts of time (50 time steps each) do higher intensity of treatment lead to a less fit tumour. As shown in Figure 1.3 (top right and top left) sometimes a smaller drug or vaccine intensity can lead to a smaller tumour fitness. Figure 1.4 shows in detail an example of this counterintuitive result. If the cost of the p53 vaccine is moderate $(0.2)$ then susceptible populations will have a chance to survive and thus to recover when the vaccine is no longer active. If this cost is increased $(0.3)$ the chemotherapy resistant population is driven beyond the point of recovery, so when chemotherapy is applied all the remaining populations are susceptible. Nonetheless, if the cost is further increased (0.4) the result is that the population that disappears during the application of the vaccine is the sensitive one whereas the chemotherapy resistant, while 
minimal, is still there. Once the treatment is switched to chemotherapy it will recover driving up the overall tumour fitness.

The model shows how the order in which the treatments are applied is important. It also suggests that the amount of time that chemotherapy is active is more important than that of the p53 vaccine. With regards to protocols where the p53 vaccine is applied first, the results suggest that treatments where the vaccines applied for longer are more effective. Although protocols that use the p53 vaccine first are usually not as effective as those that use chemotherapy first, they both have in common that the first treatment should be applied for longer than the second. This is because the role of the first treatment should be to reduce the heterogeneity of the tumour in order to diminish the chances of the tumour evolving resistance to the second treatment. Figure 1.5 highlights the importance of the first part of the treatment. Although chemotherapy prior to the p53 vaccine can yield good results, this example highlights the risk of not applying chemotherapy for long enough. In all the cases, regardless of how high the cost of chemotherapy for susceptible cells is, the tumour heterogeneity is maintained and thus little is achieved in reducing the tumour fitness.

It is no accident that we equate directly tumour fitness and tumour heterogeneity in this model. By reducing heterogeneity (i.e. in this context, knocking out one of the resistant populations) with the first treatment we can apply a suitably targeted second treatment to knock out the remaining resistant populations. Unfortunately, the reality might be that there are multiple subpopulations that would require different drug or treatment strategies to eradicate them all and of course this is simply impractical. However, if we can harness the power of strategies such as the evolutionary double bind we may exploit synergistic aspects of multiple treatments and manage to drive the tumour population to extinction even with highly heterogenous tumours.

The general model we have described here can be used to study other treatments such as radiotherapy, surgery and targeted therapies. It could also be used to study situations where resistance to a given treatment comes at a cost and where the interactions between tumour cell populations are important with regards to their susceptibility to a therapy. A key advantage of the model is its ability to quickly and easily test multiple combinations of treatments under different schedules and doses and highlight which should be chosen for experimental testing. This integrated approach to cancer treatment will not only allow for the development of potentially novel therapies but simultaneously allow them to be targeted and optimized for a given patient. Critical to this integration, however, is the feedback between model and experiment. Thus future application of this model must involve direct collaboration with experimentalists in order to properly parameterise and test model driven hypothesis.

\section{Acknowledgments}

We gratefully acknowledge the NIH/National Cancer Institute support from NCI 1U01CA151924 - 01A1, the ICBP (5U54 CA113007), PSOC (U54 CA143970-01) and TMEN (1U54 CA126505) programs.

\section{References}

1. Nowell PC. The clonal evolution of tumor cell populations. Science. 1976 Oct; 194(4260):23-28. [PubMed: 959840]

2. Gatenby RA, Brown J, Vincent T. Lessons from applied ecology: cancer control using an evolutionary double bind. Cancer Res. 2009 oct; 69(19):7499-7502. [PubMed: 19752088]

3. Anderson ARA, Weaver AM, Cummings PT, Quaranta V. Tumor morphology and phenotypic evolution driven by selective pressure from the microenvironment. Cell. 2006 Dec; 127(5):905-915. [PubMed: 17129778] 
4. Maynard Smith, J. Evolution and the theory of games. Cambridge: Cambridge University Press; 1982.

5. Tomlinson IP. Game-theory models of interactions between tumour cells. Eur J Cancer. 1997 Aug; 33(9):1495-1500. [PubMed: 9337695]

6. Gatenby RA, Vincent TL. An evolutionary model of carcinogenesis. Cancer Res. 2003 Oct; 63(19): 6212-6220. [PubMed: 14559806]

7. Basanta D, Simon M, Hatzikirou H, Deutsch A. Evolutionary game theory elucidates the role of glycolysis in glioma progression and invasion. Cell Prolif. 2008 Dec; 41(6):980-987. [PubMed: 19040573]

8. Anderson ARA, Hassanein M, Branch KM, Lu J, Lobdell NA, Maier J, Basanta D, Weidow B, Narasanna A, Arteaga CL, Reynolds AB, Quaranta V, Estrada L, Weaver AM. Microenvironmental independence associated with tumor progression. Cancer Res. 2009 Nov; 69(22):8797-8806. [PubMed: 19887618]

9. Dingli D, Chalub FACC, Santos FC, Van Segbroeck S, Pacheco JM. Cancer phenotype as the outcome of an evolutionary game between normal and malignant cells. Br J Cancer. 2009 Oct; 101(7):1130-1136. [PubMed: 19724279]

10. Basanta D, Scott JG, Rockne R, Swanson KR, Anderson ARA. The role of idh1 mutated tumour cells in secondary glioblastomas: an evolutionary game theoretical view. Phys Biol. 2011 Feb. 8(1):015016. [PubMed: 21301070]

11. Antonia SJ, Mirza N, Fricke I, Chiappori A, Thompson P, Williams N, Bepler G, Simon G, Janssen W, Lee J, Menander K, Chada S, Gabrilovich DI. Combination of p53 cancer vaccine with chemotherapy in patients with extensive stage small cell lung cancer. Clin Cancer Res. 2006 Feb; 12(3 Pt 1):878-887. [PubMed: 16467102]

12. Ramakrishnan R, Assudani D, Nagaraj S, Hunter T, Cho H, Antonia S, Altiok S, Celis E, Gabrilovich DI. Chemotherapy enhances tumor cell susceptibility to ctl-mediated killing during cancer immunotherapy in mice. J Clin Invest. 2010 Apr; 120(4):1111-1124. [PubMed: 20234093]

13. Von Neumann, J.; Morgenstern, O. Theory of games and economic behavior. Princeton: Princeton University Press; 1944.

14. Lai SL, Perng RP, Hwang J. p53 gene status modulates the chemosensitivity of non-small cell lung cancer cells. J Biomed Sci. 2000; 7(1):64-70. [PubMed: 10644891]

15. Strand DW, Franco OE, Basanta D, Anderson ARA, Hayward SW. Perspectives on tissue interactions in development and disease. Curr Mol Med. 2010 Feb; 10(1):95-112. [PubMed: 20205682] 
A
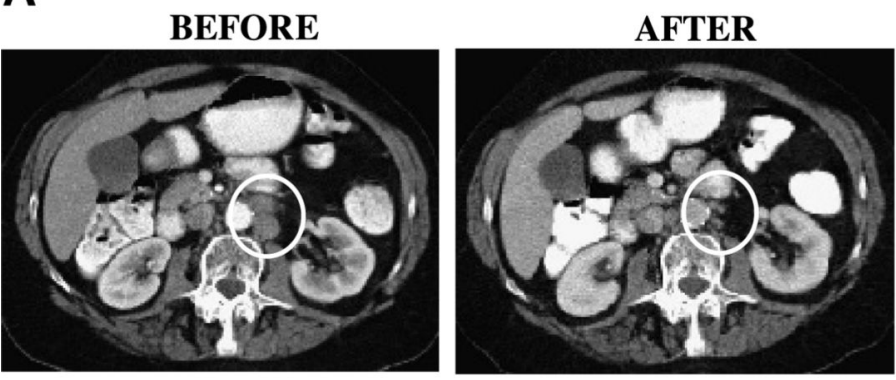

B

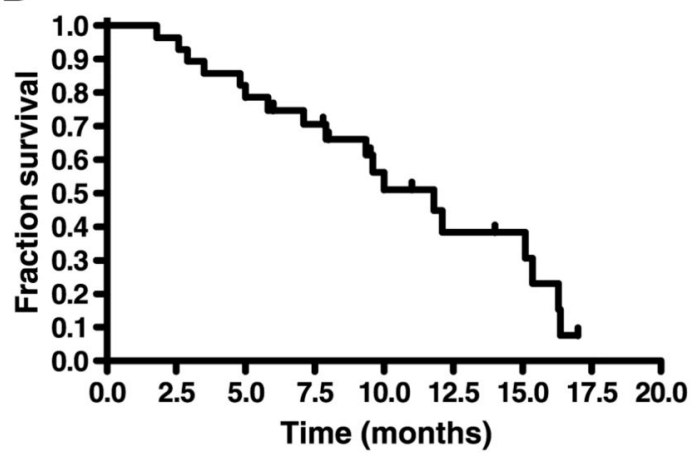

C

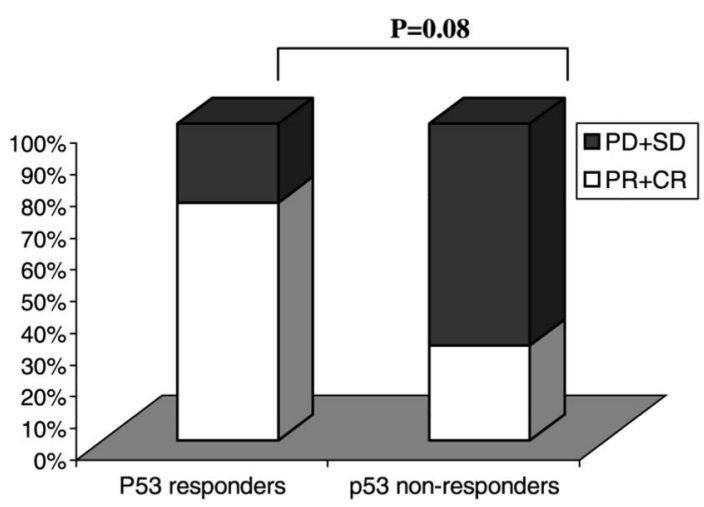

E

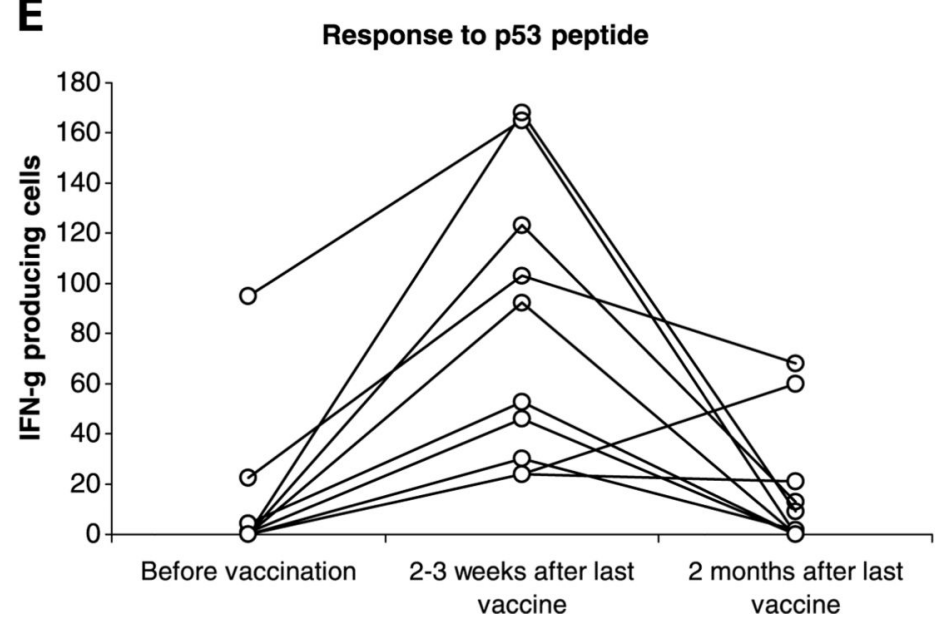

D

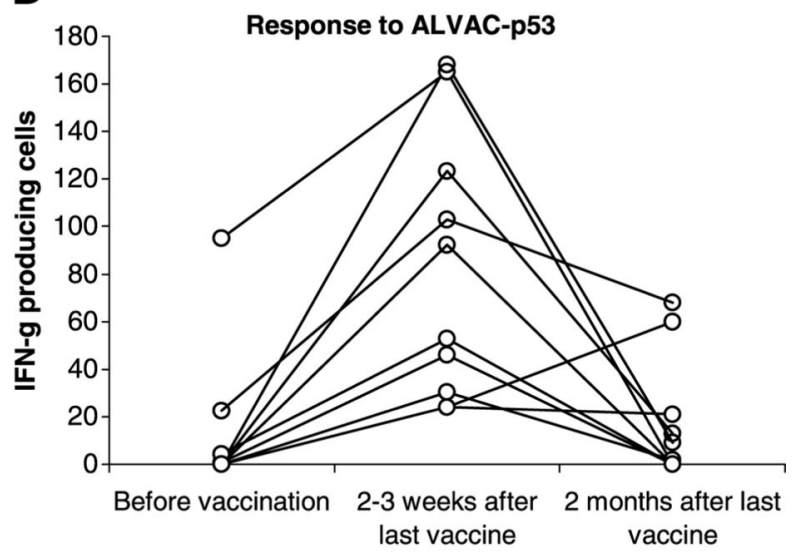

F

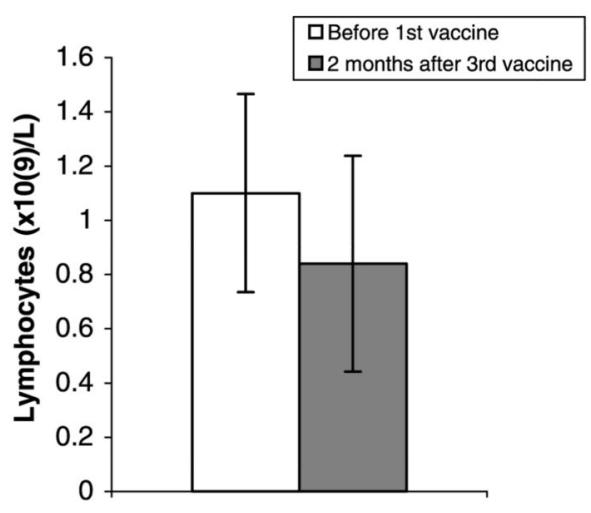

Fig. 1.1.

Experimental results show that the combination of a p53 vaccine with chemotherapy is synergistic. A. A partial response with reduction in the size of a retroperitoneal lymph node is observed by CT scans following 3 administrations of vaccine. $B$. Survival (in months) of the initially treated cohort of 29 patients from the time of the first vaccination injection $C$. More patients that were p53 immunotherapy responders showed a clinical response (PR and $\mathrm{CR}$ ) than were patients that were p53 immunotherapy non-responders. $D$, the results of IFN$\mathrm{g}$ ELISPOTassay from patients who developed p53 immune response to vaccination showing serum evidence of an immune response following the vaccine but a generally fast 
decline in immune reactivity following completion of the vaccine treatments. $E-F$. Not relevant but part of the original article from Antonia et al. [11]. 

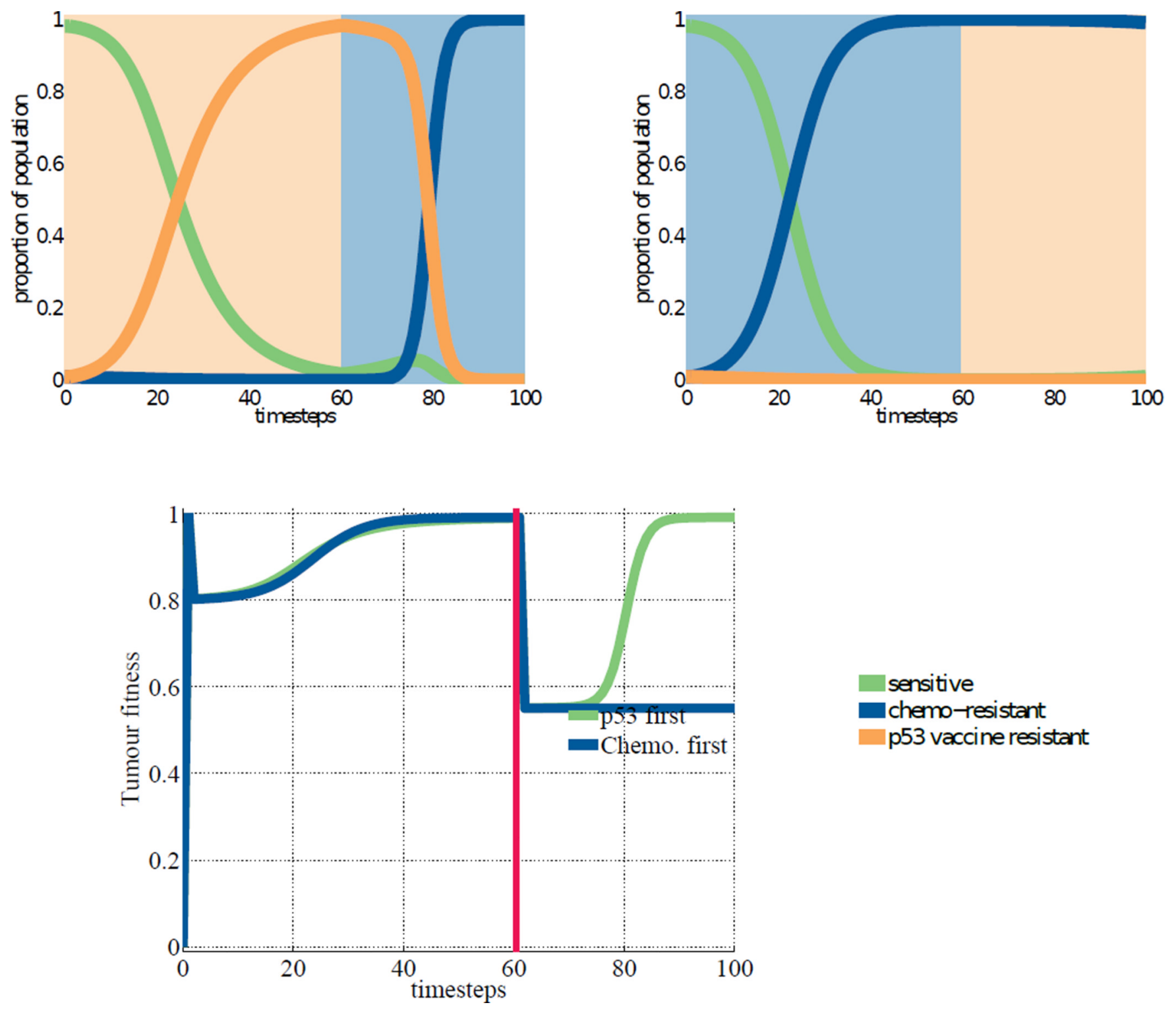

Fig. 1.2.

Comparison between a combination treatment with a p53 vaccine followed by chemotherapy and chemotherapy followed by the p53 vaccine. Both simulations chemotherapy had a cost of 0.2 and the p53 vaccine a cost of 0.4. Left Application of the p53 vaccine results in a strong selection force for tumour cells that are resistant to the vaccine. After 60 time steps the application of chemotherapy results in a strong growth for the chemotherapy resistant population which was never entirely eradicated Right The result of the same therapeutic intervention but with a different order is shown. The initially selected for chemo-resistant population leads to a situation in which the susceptible and p53 vaccine-resistant populations are virtually eradicated. Bottom The average tumour fitness under the two different orders of treatment switched at 60 time steps. 


\section{p53 vaccine first}
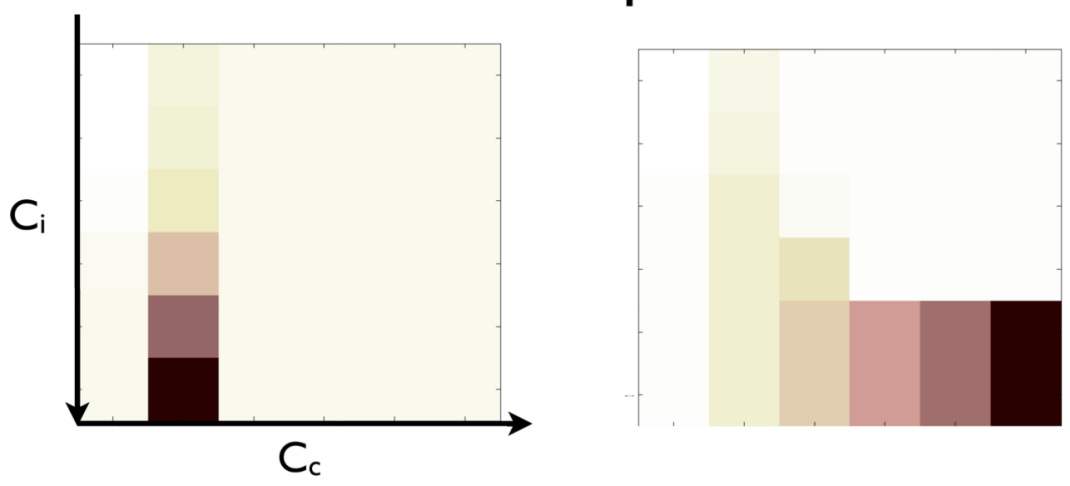

\section{Chemotherapy first}

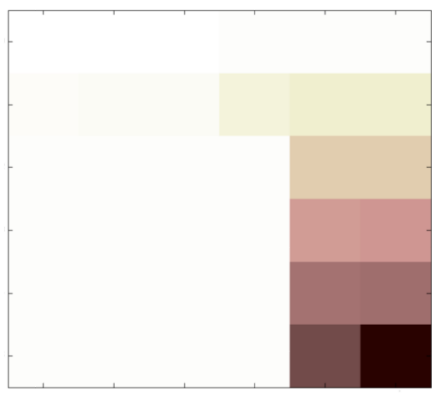

25

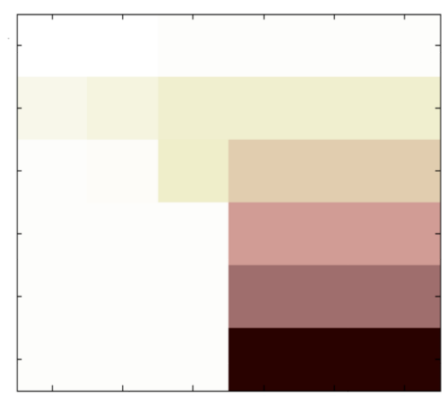

50
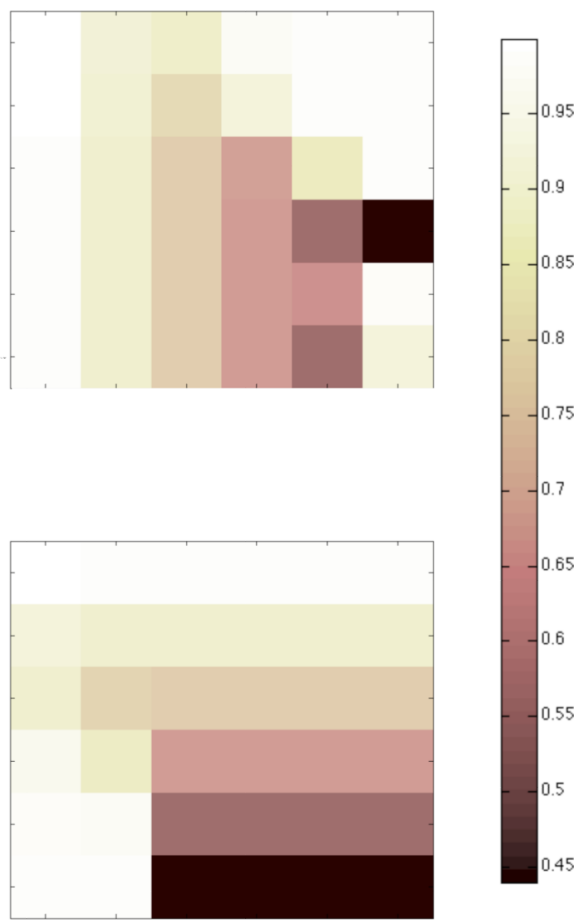

75

\section{$\%$ of time for $\left.\right|^{\text {st }}$ treatment}

Fig. 1.3.

Average tumour fitness at the end of the simulations. The following plots show the average tumour fitness (where darker shades represent less fitness) after 100 time steps in which the order, dosage and timing of the application of the two treatments is changed. The first row shows the scenarios in which the p53 vaccine is applied first whereas the second row shows the results when chemotherapy is applied first. Each column shows the effect of spending 25, 50 and 75 time steps on the first therapy (using the remain time steps for the second). Each of the grids represents the average tumour fitness after using treatments with an intensity varying between 0 and 0.5 . As an example, the box in the top left corner shows the average tumour fitness in a number of scenarios that share the fact that the p53 vaccine is applied first for 25 time steps followed by chemotherapy for 75 time steps. In this particular case the results show that increasing the cost of being susceptible to the p53 vaccine $\left(C_{i}\right)$ has a higher impact to the tumour fitness than increasing the cost of being susceptible to chemotherapy $\left(C_{c}\right)$ 

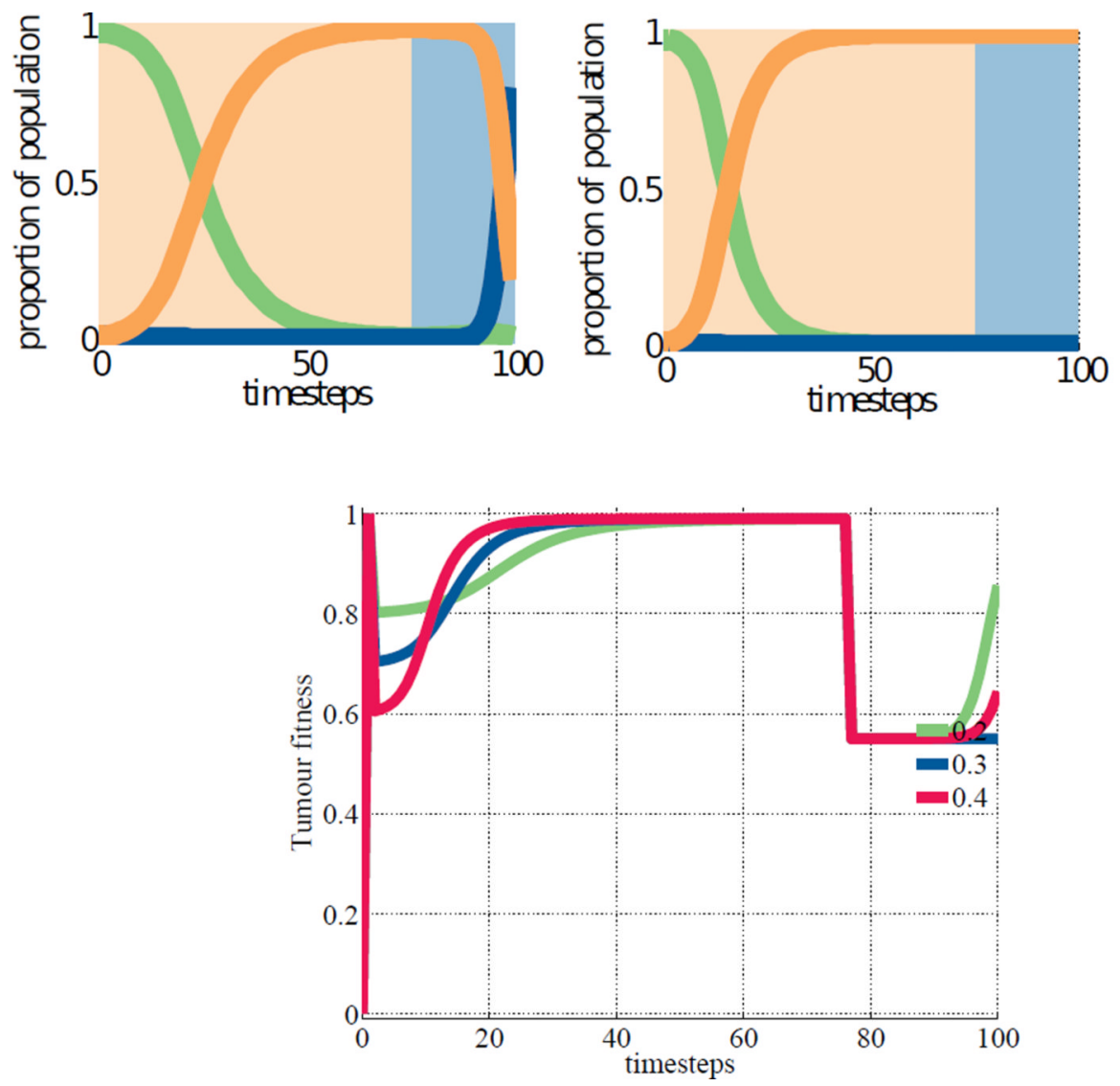
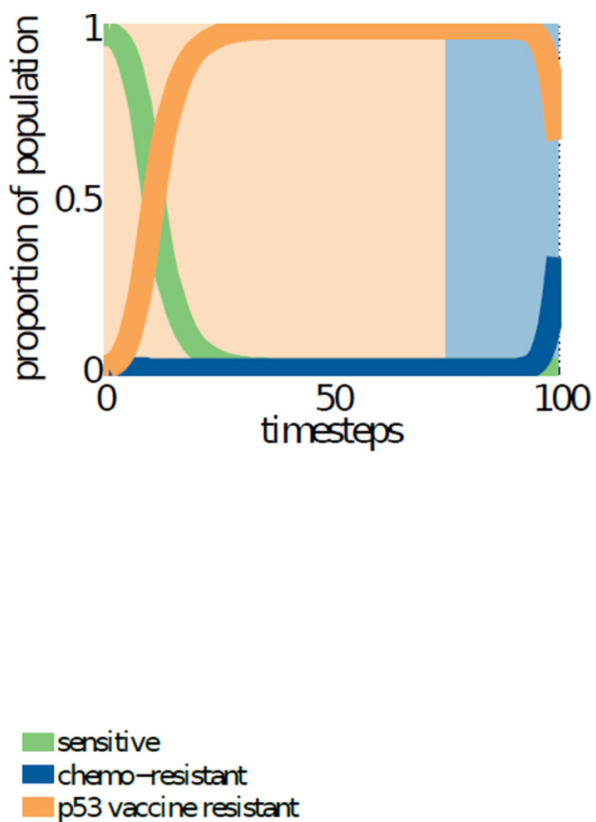

Fig. 1.4.

Three simulations where the p53 vaccine is applied first using three different penalties for cells susceptible to the p53 vaccine Left 0.2 . Application of the p53 vaccine results in a strong selection force for tumour cells that are resistant to the vaccine. After 75 time steps the application of chemotherapy results in a strong growth for the chemotherapy resistant population which was never entirely eradicated. Centre 0.3. As it was the case before, the first 75 time steps result in selection of p53 vaccine resistant cells but in this case the chemotherapy resistant population has been decimated to the extent that is unable to show any noticeable recovery when chemotherapy is applied. The original population susceptible to the two treatments, although not entirely eradicated, is unable to play a role. Right 0.4 . As it was the case previously, after the 75 time steps of p53 vaccine chemotherapy is applied. In this case the original susceptible population is entirely eradicated whereas the one that is resistant to chemotherapy, although comparatively very small, manages to survive until the application of chemotherapy results in a strong selection force for this cell type. Bottom The average tumour fitness under the three different scenarios. 

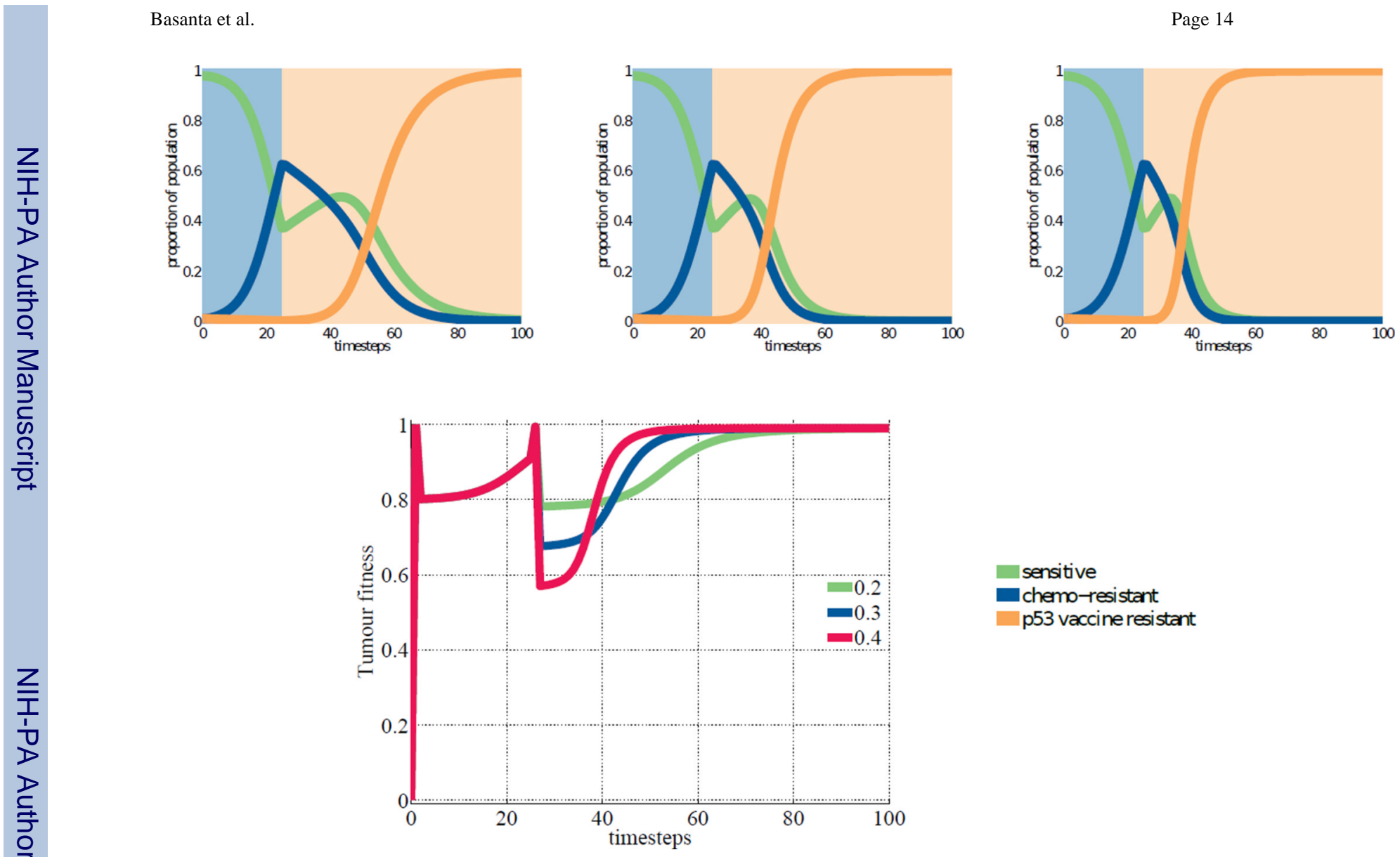

Fig. 1.5.

Three simulations where chemotherapy is applied first (0.2) using three different penalties for cells that are susceptible to the p53 vaccine. Application of chemotherapy results in a strong selection force for tumour cells that are resistant. After 25 time steps the chemotherapy resistant population has managed to grow and overtake the other two but significant amounts of sensitive and p53 vaccine resistant remain. Left 0.2. Centre 0.3. Right 0.4. Bottom The average tumour fitness under the three different scenarios. 
Table 1.1

Payoff table of the general model

\begin{tabular}{|c|c|c|c|}
\hline & $\boldsymbol{R}_{\boldsymbol{A}}$ & $\boldsymbol{R}_{\boldsymbol{B}}$ & $\mathbf{S}$ \\
\hline$R_{A}$ & $1-c_{A}-\alpha d_{B}(t)$ & $1-c_{A}-\alpha d_{B}(t)+X(t)$ & $1-c_{A}-\alpha d_{B}(t)$ \\
\hline$R_{B}$ & $1-c_{B}-\beta d_{A}(t)+Y(t)$ & $1-c_{B}-\beta d_{A}(t)$ & $1-c_{B}-\beta d_{A}(t)$ \\
\hline $\mathrm{S}$ & $1-d_{A}(t)-d_{B}(t)$ & $1-d_{A}(t)-d_{B}(t)$ & $1-d_{A}(t)-d_{B}(t)$ \\
\hline
\end{tabular}


Table 1.2

Payoff table of the p53 vaccine + chemotherapy game

\begin{tabular}{|c|c|c|c|}
\hline & $\mathbf{C}$ & $\mathbf{I}$ & $\mathbf{S}$ \\
\hline$C$ & $1-C_{c}-\alpha d_{I}(t)$ & $1-C_{c}-\alpha d_{I}(t) / 2$ & $1-C_{c}-\alpha d_{I}(t)$ \\
\hline$I$ & $1-C_{i}-\beta d_{C}(t)$ & $1-C_{i}-\beta d_{C}(t)$ & $1-C_{i}-\beta d_{C}(t)$ \\
\hline$S$ & $1-d_{C}(t)-d_{I}(t)$ & $1-d_{C}(t)-d_{I}(t) / 2$ & $1-d_{C}(t)-d_{I}(t)$ \\
\hline
\end{tabular}

